\section{Stellate nonhereditary idiopathic foveomacular retinoschisis concomitant to exudative maculopathies}

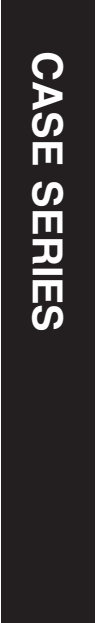

${ }^{1}$ Belfast Health and Social Care Trust and Centre for Experimental Medicine, Queen's University Belfast, Belfast, UK

${ }^{2}$ Scientific Institute San Raffaele, Vita-Salute University, Milan, Italy

Correspondence:

G Casalino, Belfast Health and Social Care Trust and Centre for Experimental Medicine, Queen's

University Belfast, Institute of Clinical Science - Block A Grosvenor Road, Belfast, Northern Ireland BT12 6BA, UK

Tel: +44 747968 7101;

Fax: +44 2890330744

E-mail: peppecasalino@ gmail.com

Received: 29 October 2015 Accepted in revised form: 4 January 2016

Published online:

26 February 2016 split along the OPL remained unaltered. Conclusions SNIFR may be associated with n-AMD. It is important to recognise the presence of retinoschisis when there is other exudative pathology as the former may be misinterpreted as intraretinal fluid, prompting unnecessary treatment.

Eye (2016) 30, 754-757; doi:10.1038/eye.2016.17; published online 26 February 2016

\section{Introduction}

Stellate foveal retinoschisis is most commonly associated with congenital juvenile X-linked

\begin{abstract}
Purpose To report the clinical course of patients presenting with stellate nonhereditary idiopathic foveomacular retinoschisis (SNIFR) concomitant with exudative maculopathies. Methods Retrospective case series. Multimodal imaging findings, including spectral-domain optical coherence tomography (SD-OCT) were reviewed. Genetic testing for the RS1 gene was performed in one patient.

Results We identified two female patients who fit the definition of SNIFR and presented with concomitant neovascular age-related macular degeneration (n-AMD). In both the patients, SD-OCT showed exudative macular features and splitting (bilateral in patient 1 , unilateral in patient 2) of the outer plexiform layer (OPL) in the macula with no other evidence of hereditary or an acquired predisposing condition. Genetic testing excluded mutation of $R S 1$ gene in patient 1 . The fundi of both the patients showed characteristic signs of active choroidal neovascularization (CNV) and following anti-VEGF treatment, visual acuity improved and $\mathrm{CNV}$-related exudative changes resolved. However, the

retinoschisis (CXLR) due to a defect in the RS1 gene. ${ }^{1}$ CXLR typically affects young men and approximately half of them also develop peripheral retinoschisis. ${ }^{1}$

Stellate nonhereditary idiopathic foveomacular retinoschisis (SNIFR) has been recently described as a new category of stellate foveal retinoschisis in which there is no evidence of hereditary or acquired predisposing condition. ${ }^{2}$ Given its recent identification, the prevalence of this condition among patients with exudative maculopathies is unknown. We retrospectively analysed the spectral-domain optical coherence tomography (SD-OCT) findings acquired on 600 new patients attending a single tertiary referral centre between May 2012 and January 2014 for exudative maculopathies in Belfast, Northern Ireland. Fluorescein angiography (FA) and indocyanine green angiography (ICGA) were available, unless clinically contraindicated.

We identified two female patients who fit the definition of SNIFR. These patients presented with neovascular age-related macular degeneration (n-AMD) and underwent anti-VEGF treatment. We herein report on multimodal imaging findings and the clinical course of these two patients.
\end{abstract}

G Casalino ${ }^{1,2}$, M Upendran ${ }^{1}$, F Bandello ${ }^{2}$ and

U Chakravarthy ${ }^{1}$

\section{Case reports}

Case 1

An 85 year-old woman was referred to our clinic for exudative maculopathy in the left eye (LE). Best-corrected visual acuity (BCVA) was 72 ETDRS letters in the right eye (RE) and 66 letters in the LE. Apart from well-controlled systemic hypertension, her medical history was unremarkable and family history was negative for any ocular disease. FA and ICGA showed an extrafoveal type 2 choroidal neovascularization 
$(\mathrm{CNV})$ in the LE and scattered reticular pseudodrusen at the posterior pole of both eyes were visible on blue FAF (Figures 1 and 2). SD-OCT scans revealed intraretinal fluid (IRF) in the LE and also the presence of foveomacular retinoschisis in both eyes (Figures 1 and 2); no peripheral retinoschisis was seen on the fundus examination of both eyes. The patient was treated with intravitreal injection of ranibizumab $(0.5 \mathrm{mg}$ per $0.05 \mathrm{ml})$ with a loading phase of monthly treatments over a 3- month period to the LE. One month after the third injection, BCVA slightly improved to 70 letters in the LE and was unchanged in the RE; SD-OCT scan showed resolution of IRF in the LE. However, the foveomacular retinoschisis remained unaltered (Figure 1). Two years after the initiation of treatment, the OCT scans showed no recurrence of CNV activity and the vision in the LE was 69 letters (Figure 1). No pathogenic mutations were detected within the coding sequence of RS1 gene.

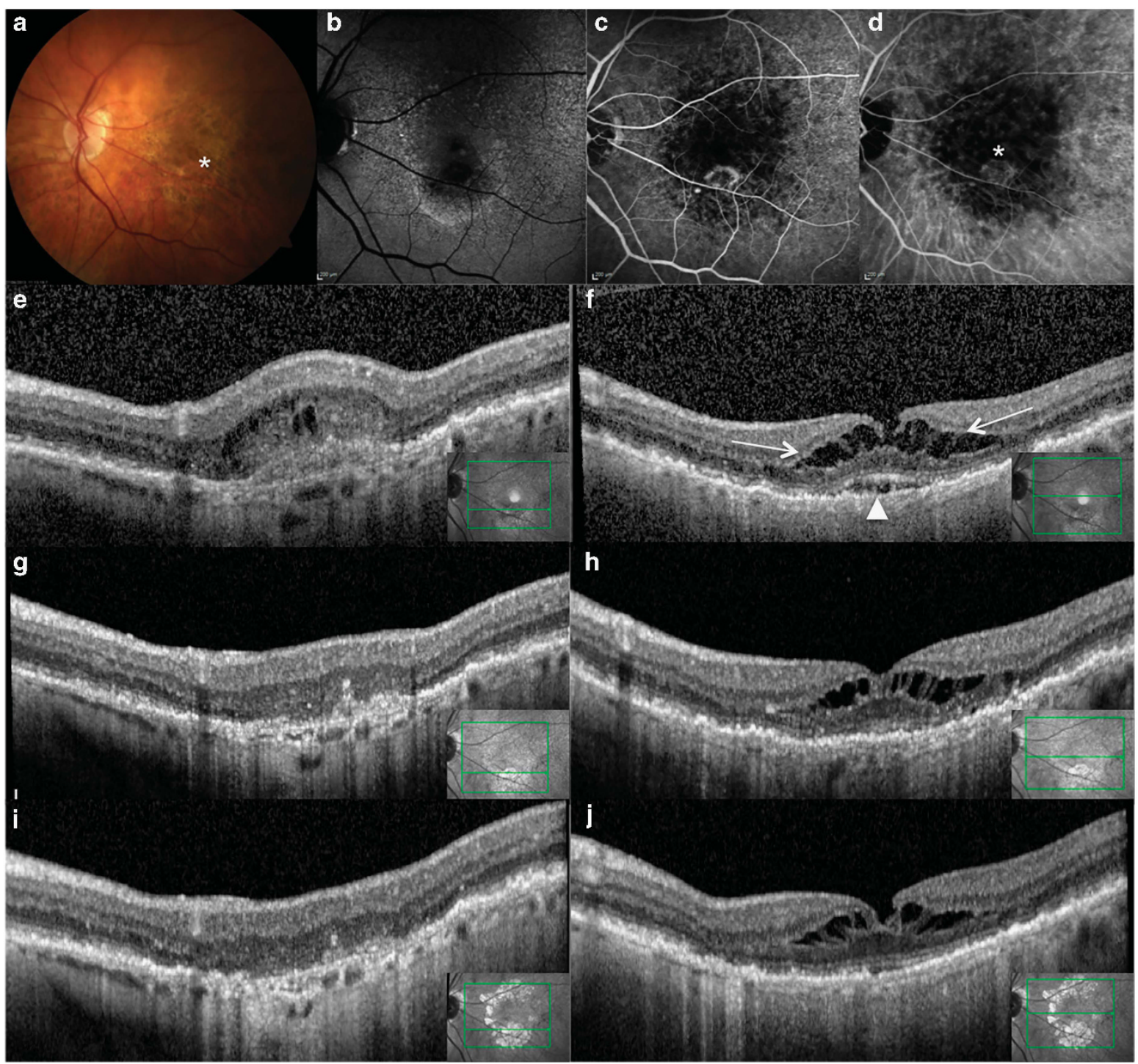

Figure 1 Case 1. Multimodal imaging of left eye. At presentation, colour fundus photograph (CFP) (a) shows a slight swelling of the retina underneath the fovea (arrows). Blue fundus autofluorescence (FAF) (b) shows diffuse RPE disturbance and reticular pseudodrusen. Fluorescein angiography (FA) frame (c) shows a well-defined hyperfluorescence and the corresponding indocyanine green angiography (ICGA) (d) shows a neovascular net consistent with a type 2 choroidal neovascularization (CNV; asterisk). Optical coherence tomography (OCT) scan in correspondence of the CNV (e) shows subretinal hyperreflective material (HRM) and intraretinal fluid (IRF); the same scan 3 months (g) and 24 months (i) after the initiation of anti-VEGF injections shows resolution of HRM and IRF. OCT scan in correspondence of the fovea at presentation (f) shows a shallow pigment epithelial detachment (PED; arrowhead) and foveomacular retinoschisis with characteristic splitting of the outer plexiform layer (OPL; arrows); the same scan 3 months (h) and 24 months (j) after the commencement of treatment showed resolution of PED and unchanged retinoschisis. 


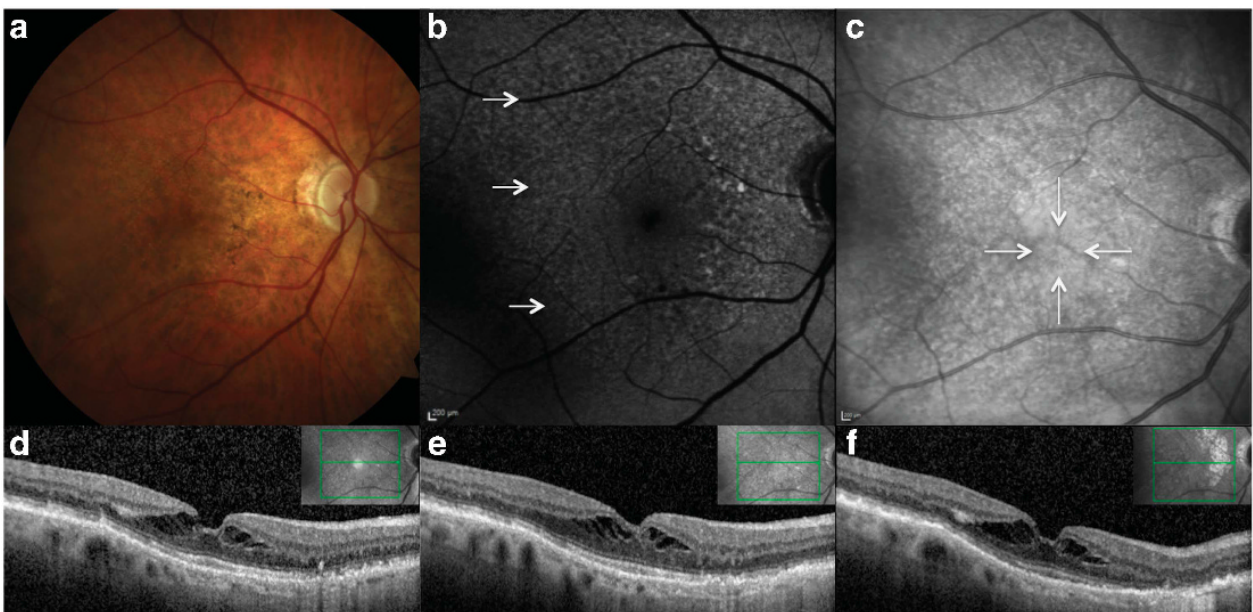

Figure 2 Case 1. Multimodal imaging of right eye. CFP (a) shows pigment abnormalities and drusen; blue FAF (b) shows diffuse reticular pseudodrusen (arrows); infra-red reflectance photograph (c) barely shows radial spoking around the fovea (arrows). OCT scans at presentation (d), at 3 months (e), and at 24 months (f) of follow-up, show splitting of the OPL at the macula.

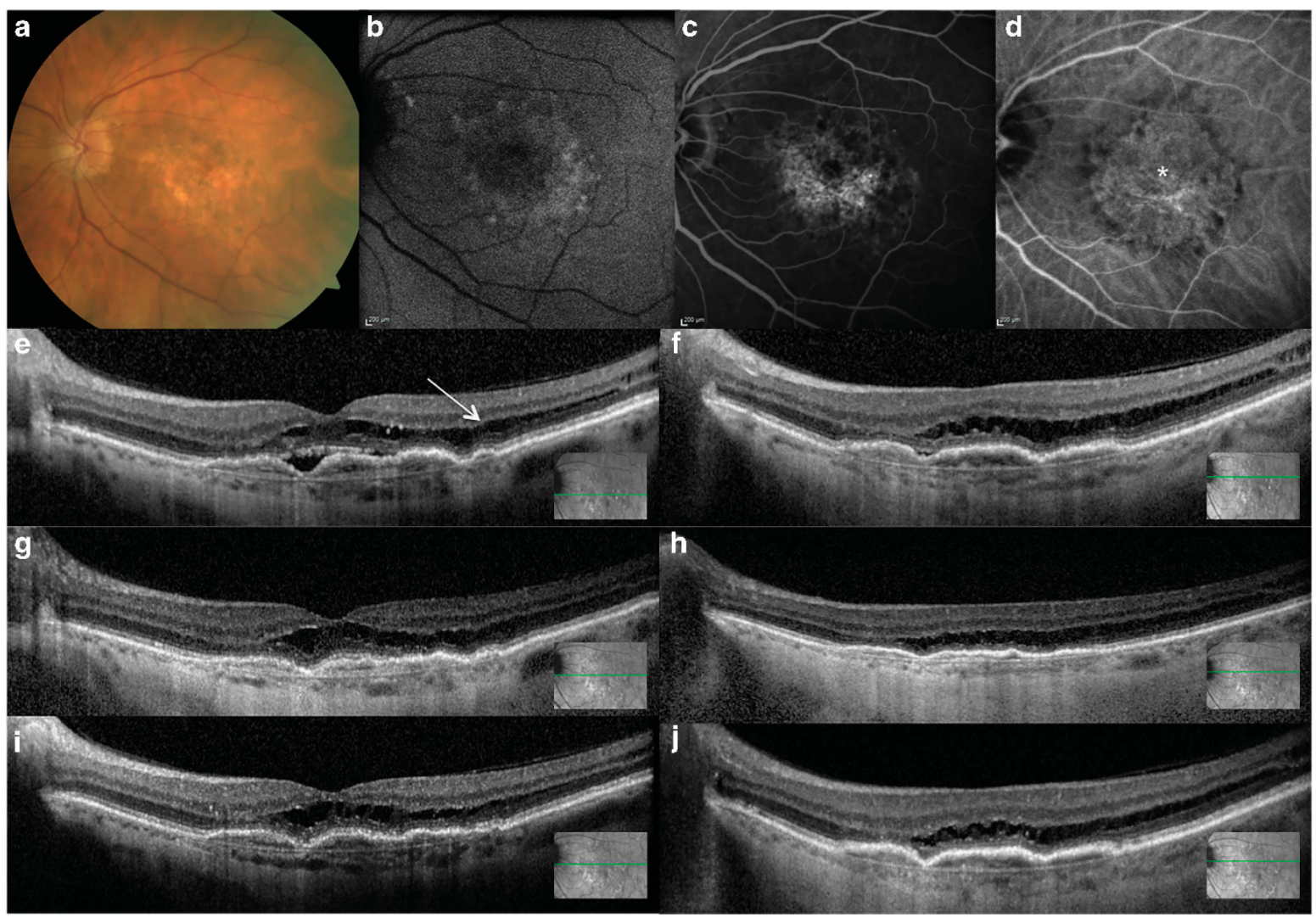

Figure 3 Case 2. Multimodal imaging of the left eye. CFP (a), FA (c), and ICGA (d) show a large occult CNV (asterisk) with no evidence of reticular pseudodrusen on blue FAF (b). OCT scan in correspondence of the fovea shows subretinal fluid (SRF), a shallow PED, and a foveomacular retinoschisis with characteristic splitting of the OPL (arrows) at presentation (e); 3 months (g) and 12 months (i) after the initiation of anti-VEGF injections, SRF is no more detectable and retinoschisis is still evident. OCT scan passing above the fovea shows a more evident and extensive splitting of the OPL, which remained stable from the baseline (f), to 3 months (h), and to 12 months (j) after the treatment. 


\section{Case 2}

An 81 year-old woman came to our attention for exudative maculopathy in the LE. BCVA was counter finger in RE and a disciform scar was found to be present in this eye. BCVA in the fellow eye was 66 letters. She had been a heavy smoker and she suffered from chronic obstructive pulmonary disease. Family history was negative for any ocular disease. FA and ICGA showed the presence of a type $1 \mathrm{CNV}$ and concomitant presence of foveomacular retinoschisis, as revealed by SD-OCT scan (Figure 3). Peripheral retinoschisis was absent on the fundus examination of both eyes. The patient underwent three monthly intravitreal injections of aflibercept $(2.0 \mathrm{mg}$ per $0.05 \mathrm{ml}$ ) to the LE. One month after the third injection, BCVA slightly improved to 70 letters in the LE. SD-OCT scan showed no signs of neovascular activity in the LE and the foveomacular retinoschisis seemed to be not affected by the intravitreal injections (Figure 3). Twelve months after the initiation of treatment, the OCT scans showed no recurrence of CNV activity and the vision in the LE was 71 letters.

\section{Discussion}

Recently Ober et $a l^{2}$ described a series of patients with idiopathic retinoschisis. In this series, there was female predominance (16 females and one male) and only two patients presented bullous peripheral retinoschisis in addition to the typical foveomacular retinoschisis. Genetic testing for RS1 gene was performed in eight patients and revealed no known disease-causing mutations. All the patients demonstrated splitting of the outer plexiform layer (OPL) in the macula and the visual acuity was relatively preserved, except in a single patient in whom subretinal fluid developed under the fovea.

The aim of our study was to see whether this condition occurred in patients diagnosed as exudative maculopathy undergoing intravitreal injections. We excluded patients presenting with retinoschisis secondary to epiretinal membrane, vitreomacular traction, myopia, and other acquired predisposing conditions. On a total of 600 patients analysed, we found two patients $(0.3 \%)$ with idiopathic retinoschisis with n-AMD. The patients were females and there was no family history of CXLR or other hereditary maculopathies. The absence of peripheral retinoschisis, the female gender, and the absence of mutation in RS1 gene in case 1 were in keeping with those reported by Ober et $a l^{2}$ in their series.
The typical stellate foveomacular appearance at the macula of our patients was not present. We believe this was possibly due to the concomitant presence of AMD. However, in the RE of case 1, in which there was no exudative maculopathy, infra-red reflectance imaging barely showed the stellate appearance with radial spoking around the fovea.

As far as we know, this is the first report on the association of foveomacular retinoschisis with n-AMD. In our cases, intravitreal anti-VEGF injections were effective in that treatment resulted in resolution of the exudative maculopathy but the retinoschisis remained unaffected. In summary, SNIFR and exudative maculopathies can occur in the same eye. It is important to recognise the presence of foveal retinoschisis when concomitant to a neovascular complex, because retinoschisis can be misinterpreted as intraretinal fluid, possibly prompting unnecessary treatment.

\section{Summary}

\section{What was known before}

- Stellate nonhereditary idiopathic foveomacular retinoschisis (SNIFR) is a new category of retinoschisis.

- In SNIFR, visual acuity is relatively preserved.

What this study adds

- SNIFR may be concomitant to exudative maculopathies, such as neovascular age-related macular degeneration (n-AMD) in which vision may be compromised.

- In patients with exudative maculopathies, multimodal imaging is useful to recognise foveal retinoschisis that may be misinterpreted as intraretinal fluid, prompting unnecessary treatment.

\section{Conflict of interest}

The authors declare no conflict of interest.

\section{References}

1 Molday RS, Kellner U, Weber BH. X-linked juvenile retinoschisis: clinical diagnosis, genetic analysis, and molecular mechanisms. Prog Retin Eye Res 2012; 31: 195-212.

2 Ober MD, Freund KB, Shah M, Ahmed S, Mahmoud TH, Aaberg TM et al. Stellate nonhereditary idiopathic foveomacular retinoschisis. Ophthalmology 2014; 121: 1406-1413. 